\title{
CASE
}

OF

\section{AXILLARY ANEURISM}

SUCCESSFULLY TREATED

BY TYING THE

\section{SUBCLAVIAN ARTERY.}

By CHARLES ASTON KEY, Eso.

SURGEON TO GUY'S HOSPITAL.

Read Nov. 11 th, 1823.

George VAughan, æt. 36, whose occupation is that of an extra-tide waiter at the Custom House, in the month of July last, while making a sudden exertion with his right arm, felt (as he expressed it) something snap below the collar bone, attended with a slight degree of pain. In a day or two afterwards, he perceived a small tumor on the fore part of the chest, about an inch and a half below the clavicle, which pulsated strongly. It continued to increase in size, but was not attended with much pain or inconvenience, nor was he induced to refrain from his usual occupation.

On the 29th of August, he presented himself at Guy's Hospital as an out patient, and I detained him for the opinion of Sir Astley Cooper. The 
aneurism was large, forming a regular circumscribed swelling from the edge of the clavicle to the lower edge of the pectoral muscle; the pulsation was strong, and could be felt distinctly in all parts of the sac; his arm was not painful nor swelled, but he complained of slight numbness in his fingers. His health was unimpaired, and he appeared in all respects in a condition to benefit by an operation. It should likewise be mentioned, that pressure on the subclavian artery not only commanded the pulsation in the aneurismal sac, but so effectually prevented the blood entering the sac, as to empty it almost entirely of its fluid contents. The nature of his complaint was explained to him; and he left the hospital with injunctions, to keep his arm quiet, to live temperately, and, if possible, to relax in his attendance on his usual employment.

He called on me once or twice with the aneurism and the limb precisely in the same state. As I was about to leave town for a few days, I did not propose the operation to him immediately; but cautioned him against using, or allowing to be used, any means that the officious ignorance of friends might suggest.

In a day or two after my return, he again called on me; and surprised me by the visible alteration in his appearance. His countenance was pallid, and covered with a cold perspiration, 
and indicated great distress; his arm and hand were extremely œdematous; the subcutaneous veins of his arm, shoulder, and side of the neck, were distended with blood, and formed a varicose plexus, evincing the degree of pressure, under which the axillary vessels were labouring. The aneurismal tumor had lost its pulsation, and could not be felt to vibrate from the ingress of the blood; it had increased considerably in size, extending lower down into the axilla, and forming a more prominent tumor under the pectoralis major. The pain he experienced, was unceasing, depriving him of rest, and producing a high degree of constitutional irritation; his pulse above a hundred in a minute, and sharp; his tongue white and glassy. The pain was most intense along the inner side of his arm, extending from the back part of the axilla to his elbow. He then explained the cause of this change; during my absence, in consequence of some uneasy sensations he began to experience in the shoulder, he was recommended by a surgeon to try the effects of pressure in reducing the tumor; for this purpose a piece of cork was bound over the artery above the clavicle, and bandages with pads were applied tightly about the tumor: the axillary vessels and plexus of nerves unable to bear this pressure, resented such treatment by producing the ill effects above mentioned.

After I had explained to him the nature, and 
the urgency of his symptoms, and that the only. means of alleviating his sufferings consisted in the operation of securing the artery by ligature, he expressed his willingness to come into the hospital, and to submit to any operation that could hold out a chance of relief. On Thursday, September 19th he was admitted into the hospital; and Mr. Travers coinciding in the opinion, that the operation of tying the subclavian artery afforded the probability of being attended with success, one o'clock on the following day was appointed for its performance.

The patient being brought into the operating theatre, the extent of the sac in the neighbourhood of the subclavian artery was ascertained, to guard, if possible, against the danger of opening it during the operation of passing the ligature under the vessel; it appeared to be bounded above by the subclavian muscle, and the artery above the clavicle appeared (as far as the finger could ascertain) to be healthy. The clavicle was raised to its utmost, and was curved considerably backward towards the trapezius muscle; it could not be raised higher by pressure made upwards against the elbow. The patient being laid upon an inclined plane, so that the light from a large skylight might be thrown into the triangular space in which the artery lies imbedded, I began the external incision in the following manner. Standing by the patient's right side, I drew the integuments 
down over the clavicle with my left hand; and cut freely upon the bone, beginning about half an inch over the clavicular portion of the sternomastoid, and continuing the incision outwards for three inches. The integuments being relaxed, the incision became raised about the third of an inch, above the clavicle, and exposed a strong platysma myoides, which was divided to the same extent. Numerous turgid veins were now exposed lying upon the cervical fascia; to avoid them was impossible; they were therefore divided, and about three ounces of blood were quickly lost; one larger than the rest was secured by Mr. Travers to prevent any obstruction from hemorrhage in the after-steps of the operation. The dense outer layer of the cervical fascia was then more freely divided, and the loose cellular texture enveloping the glands of the neck being detached by the finger, the omo-hyoideus was laid bare. A little farther dissection with the end of a director exposed the artery to the finger, pulsating over the rib; but the depth of the angle, in which it was inclosed, rendering it impossible to pass a ligature under it, about three quarters of an inch of the clavicular portion of the sterno-mastoid was divided, which afforded sufficient room, and rendered the concluding part of the operation easy; the artery became readily exposed to view, and an armed aneurismal needle was passed with facility under it. A single ligature of silk was tightened around the vessel, and the edges of the 
wound brought into contact with two sutures and adhesive plaster. The patient had been so little exhausted by the operation, which lasted twenty minutes, that he expressed a wish to walk down stairs to his bed, which of course was not consented to.

The absence of any very untoward symptoms renders it unnecessary to trouble the Society with a detail of the conclusion of the case. It will be sufficient to mention, that eight and forty hours after the operation, a general excitement of the system with an acceleration of pulse induced $\mathrm{Mr}$. Travers to prescribe (during my absence from town) a purgative of scammony and calomel, which not only removed the symptoms of general fever, but also a retention of urine, arising from a slight stricture, under which he had previously laboured. His urine had been twice drawn off by the introduction of the catheter. A trivial irritation of the trachea, inducing an occasional cough with expectoration, was allayed by the opiate linctus of the hospital. Opiates in other forms were not found necessary, with the exceptions of two nights on which his rest had been broken, when gtt. xxv tinct. opii were given him. The œdema of the limb subsided quickly after the ligature was applied to the vessel; its natural warmth was maintained, perhaps aided by being enveloped in a double fold of thin flannel; and the pain of which he complained prior to the 
operation, altogether left him as soon as he returned to his bed. The local treatment consisted in the application of a light poultice to the wound, after the removal, on the fourth day, of the adhesive straps and sutures, and in the prevention of sinuses from the lodgment of purulent matter.

The ligature was found lying detached in the wound, on Wednesday morning October 1st, the twelfth day after its application, and was removed without hemorrhage. The wound was nearly cicatrized, with the exception of the aperture occasioned by the ligature, which is gradually closing. On the following day he was allowed to leave his bed, and walk about the ward, to relieve the fa. tigue occasioned by the uniform position he had scrupulously maintained, till the ligature came away. The tumor is gradually subsiding, gives him no uneasiness, and promises to be absorbed without inflammation or suppuration. The pulse at the wrist cannot yet be felt.

It remains for me to acknowledge the kind assistance afforded me by Mr. Travers, not only during the operation, but also in the after treatment of the case, together with the interest he manifested in its progress. The wound was attended with the greatest care by Mr. Herbert of Farringdon, Berks, at that time a dresser to Sir Astley Cooper, to whose assiduity the patient is indebted for its speedy cicatrization. 
The fatality hitherto attending the operation in the practice of this town, has induced me to lay this case before the Society, as a proof, in addition to cases published by surgeons of other countries, that we may expect as much success to attend the ligature upon the subclavian artery, as upon the other large arteries of the body. Past experience may perhaps not appear to warrant this expectation; but in estimating the probability of success, we are to regard not so much the number of fatal cases, as the causes of this fatal termination; and whether they are adventitious or essential to the operation of the ligature on this vessel. The only circumstance, that ought to deter a surgeon from putting a ligature around any artery in the human body (I will not except the aorta) is, the uniform cessation of circulation in the parts below the ligature. That this is a rare occurrence after the subclavian operation, surgical records will prove; within my own experience, the operation has been performed three times without the least tendency to that state. The cause of death must therefore be sought for in circumstances not necessarily connected with the operation, and which may consequently be avoided. The most common causes of death, I believe, will be found to be, suppuration in the anterior mediastinum, in consequence of the violence done to the parts during the operation; and inflammation of the pleura, with consequent effusion into the cavity of the thorax. The former may be attributed 
solely to the injudicious disturbing of the reticular membrane connecting the artery with the scalenus anticus and with the pleura. This reticular texture will be found to be continuous with a similar texture in the anterior mediastinum, and inflammation set up in the former quickly spreads through the latter, in consequence of the free communication of the cells. In performing the operation the surgeon may altogether avoid this danger, by confining the point of his director or aneurismal needle to that part of the artery which lies upon the rib; the pulsation is here more readily felt, the situation of the vessel more easily ascertained, and the artery is more accessible to the ligature, than where it is emerging from behind the scalenus muscle. Inflammation of the pleura is to be apprehended, more from the advanced state of the disease prior to the operation, than from injury done during its performance. The extent of the sac, which quickly attains a great size in consequence of the loose texture of the cellular membrane under the pectoral muscle, causes great local irritation by its pressure on the axillary nerves, and the effect of irritation upon a nerve in producing inflammation in the part to which it is distributed, is happily illustrated by Mr. Brodie's experiment on the par vagum. By its pressure on the neighbouring veins, the sac also induces a general œdematous effusion, in which the pleura often partakes; and from the peculiar termination of the bronchial veins on the left side of the body, serous effusion from the pleura 
pulmonalis, when the disease exists on that side, may be regarded as a probable occurrence. In a patient, on whom I performed the operation in the winter of 1822, and who lived till the seventh day, the appearances I have alluded to were present, and the pericardium was highly inflamed and lined with a coat of adhesive matter.

I shall conclude this paperby adverting to two circumstances, which greatly facilitate the operation ; one, is the division of part of the clavicular portion of the sterno-mastoid muscle, to which I should not again have alluded, had it not been expressly condemned by some of our best writers. The other circumstance which is equally important, is the mode of conducting the needle under the artery, and of raising the end of the ligature from the eye of the needle to the surface of the wound. I believe this to have constituted the principal difficulty in every operation on this artery since Mr. Ramsden's case at St. Bartholomew's hospital. I have therefore subjoined a drawing of the needle and ligature I employed on this occasion, and I believe that it will be found useful in all cases, in which, from the depth of the vessel, the extremity of the needle necessarily eludes the eye of the operator.

The needle* consists of a piece of iron fixed in a

* The needle is one used by Sir Astley Cooper, but with the curve altered. 
fat ivory handle, and the curve that $I$ have found most manageable in operating upon the larger arteries is represented. After passing the silk through the eye of the needle, a common loop and knot are made at $a$; the ends of the ligature being drawn tight so as to bring the knot close to the eye of the needle, a second knot is made at $b$; by pulling the second knot, the knot $a$ can be kept close to the eye of the instrument. The operator, having passed the needle under the artery, feels for the extremity of the instrument and the knot drawn close to its eye; by gently moving the knot, the loop becomes disengaged, and can be drawn to the surface of the wound. Both knots being cut off, the operator can use one or two ligatures, as he may deem expedient.

Since the communication of this case to the Society, the patient has been gradually gaining the use of his arm, which is now nearly as perfect as that of the other limb; the tumor has disappeared, with the exception of a hardness which usually remains after the absorption of an aneurismal tumor. The pulse at the wrist I have not been able to discover, though others, who have examined the limb, have felt something like a thrill in the situation of the radial artery.

May, 1824. 


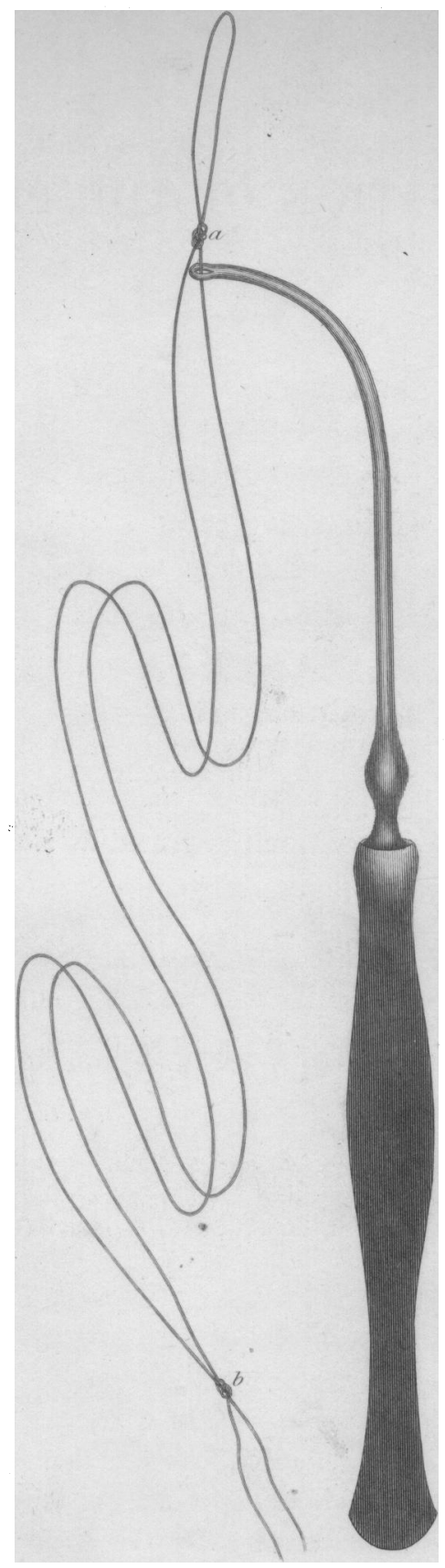

Bubl by Longmem \& 1 . Angt 1825 